\title{
Floer Homology and Homotopy Planes
}

\author{
Stefan Schröer \\ e-mailschroeer@mathematik.uni-bielefeld.de \\ fax 49 (0)521 1064743
}

\begin{abstract}
We compute the Floer homology of a certain family of homology 3spheres occuring as the boundary of a compactification of complex homotopy planes. The Floer complex turns out to be concentrated in even degrees and 2-periodic. This is proved by using suitable bordisms to Seifert fibred spaces.
\end{abstract}

\section{Introduction}

A homology plane is a smooth 2 -dimensional variety over $\mathbb{C}$, such that its associated analytic space is acyclic. A homotopy plane is a homology plane whose associated analytic space is contractible.

Recently homology planes played some role in the computation of Donaldson polynomials by Fintushel and Stern, using a topological cut and paste technique called rational blow-down $([\mathrm{St}])$.

The classification problem for homology planes is solved in [tD-Pe] for homotopy planes of logarithmic Kodaira dimension 1. These homotopy planes are distinguished by their minimal compactification divisor. The set of algebraic isomorphism classes of such homotopy planes is parametrised by integers $(a, b, m)$ with $a, b$ relatively prime, $1<a<b$ and $0<m$. Let $P(a, b, m)$ be the corresponding homotopy plane. By $[\mathrm{Ra}]$ a closed regular neighborhood of a compactification divisor in a suitable algebraic compactification of $P(a, b, m)$ is bounded by a closed smooth oriented 3-manifold $S(a, b, m)$, whose diffeomorphism type is uniquely determined by $P(a, b, m)$. Since $P(a, b, m)$ is contractible, the 3-manifold $S(a, b, m)$ is a homology 3 -sphere. Our main theorem is:

Theorem 1.1 The Floer group $\operatorname{HF}(S(a, b, m))$ of the homology 3-sphere $S(a, b, m)$ is a free abelian group of rank $\frac{1}{3} m\left(a^{2}-1\right)\left(b^{2}-1\right)$, lying in the even part of the grading. It is 4-periodic. If $a, b$ are odd it is 2-periodic.

Our proof is inspired by [St-Sz], where similiar calculations have been made in the special case $a=2, m=1$. 
Acknowledgement: I would like to thank Prof. Bauer for directing me to this problem.

\section{Descriptions of the homology 3-spheres}

In [tD-Pe] it is shown that the homotopy plane $P(a, b, m)$ allows a smooth algebraic compactification such that the compactification divisor $D(a, b, m)$ has smooth rational irreducible components and normal crossings with intersection graph

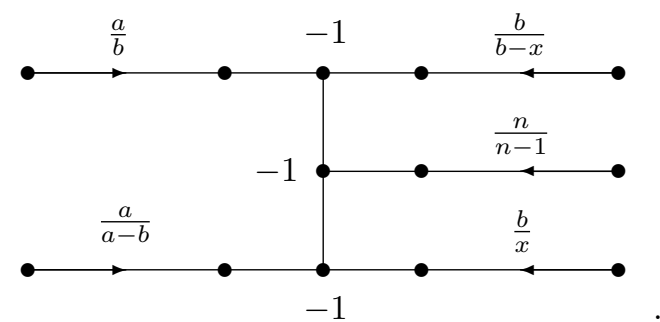

Here $x$ is defined by $-x \equiv a \bmod b, 0<x<b$. The symbol

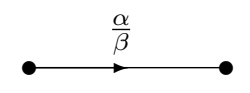

means

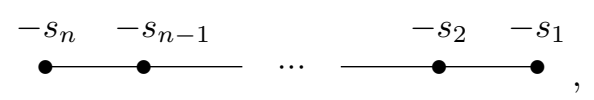

where the $s_{i}>1$ are uniquely defined by the continued fraction development

$$
\frac{\alpha}{\beta}=\left[s_{n}, \ldots, s_{1}\right]:=s_{n}-\left[s_{n-1}, \ldots, s_{1}\right]^{-1},[]:=\infty .
$$

A tubular neighborhood of the divisor $D(a, b, m)$ is a plumbing of $D^{2}$-bundles over 2 -spheres according to the $\mathbb{Z}$-weighted graph (2.1), whose boundary $S(a, b, m)$ is diffeomorphic to a Dehn surgery on a framed link in $S^{3}$ associated to the $\mathbb{Z}$ weighted graph $(2.1)$.

Let $\bar{Q}:=\mathbb{Q} \cup\left\{\frac{1}{0}\right\}$. To each $\overline{\mathbb{Q}}$-weighted tree $(\Gamma, w)$ we associate a $\overline{\mathbb{Q}}$-weighted unoriented link $L=L(\Gamma, w)$ in $S^{3}$ : Each vertex $v$ of $\Gamma$ contributes an unknot $L_{v}$ with weight $w(v)$, such that for each edge $\left\{v, v^{\prime}\right\}$ of $\Gamma$ the knots $L_{v}$ and $L_{v^{\prime}}$ link each other geometrically once.

We collect some necessary remarks on Dehn surgery. For us the term "diffeomorphism" between oriented smooth manifolds always means orientation preserving diffeomorphism unless otherwise stated. Let $M$ be an oriented smooth 3 -manifold. Let $K$ be an unoriented knot in $M$. We also write $K$ for the image 
of $K$ in $M$. Let $\nu K$ be a tubular neighborhood of $K$. Orient $\partial \nu K$ as the boundary of $\nu K$ ("outward normal vector first"). A framing of $K$ is an isotopy class of diffeomorphisms $f: S^{1} \times S^{1} \rightarrow \partial \nu K$. A framed knot $(M, K, f)$ yields a diffeomorphism class of an oriented 3-manifold $M(K, f)$, the surgery on $(M, K, f)$, defined as the colimit over the diagram

$$
M \backslash \nu K \hookleftarrow \partial \nu K \stackrel{f}{\longleftarrow} S^{1} \times S^{1} \hookrightarrow D^{2} \times S^{1}
$$

The set $\operatorname{Fr}(K)$ of all framings of $K$ is a principal right torsor of $\pi_{0} \operatorname{Diff}\left(S^{1} \times S^{1}\right)$. Let $[f]$ denote the orbit of $f \in \operatorname{Fr}(K)$ under the induced action of $\pi_{0} \operatorname{Diff}\left(D^{2} \times\right.$ $\left.S^{1}\right)$ via

$$
0 \rightarrow D_{\infty} \simeq \pi_{0} \operatorname{Diff}\left(D^{2} \times S^{1}\right) \stackrel{\partial}{\longrightarrow} \pi_{0} \operatorname{Diff}\left(S^{1} \times S^{1}\right) \simeq \operatorname{SL}(2, \mathbb{Z}),
$$

where $D_{\infty}$ is the infinite dihedral group, identified with the upper triangular matrices in $\mathrm{SL}(2, \mathbb{Z})$. By the universal property of colimits, $M(K, f)$ depends only on $[f]$. Now let $M$ be an oriented homology 3-sphere. Choose an orientation for $K$. Then $K$ is equipped with a canonical framing $f_{0}$ defined by

$$
S^{1} \times 0 \mapsto \text { meridian, } 0 \times S^{1} \mapsto \text { longitude of } K \text {. }
$$

Since

$$
\pi_{0} \operatorname{Diff}\left(S^{1} \times S^{1}\right) / \pi_{0} \operatorname{Diff}\left(D^{2} \times S^{1}\right) \simeq \mathrm{SL}(2, \mathbb{Z}) / D_{\infty} \simeq \overline{\mathbb{Q}}
$$

by

$$
\mathrm{SL}(2, Z) \ni\left(\begin{array}{ll}
x & y^{\prime} \\
y & x^{\prime}
\end{array}\right) \mapsto \frac{x}{y} \in \overline{\mathbb{Q}},
$$

each $\frac{x}{y} \in \overline{\mathbb{Q}}$ defines an orbit $f_{0} \cdot \frac{x}{y}$ in $\operatorname{Fr}(K)$ independent of the orientation of $K$, and thus defines an oriented 3-manifold $M\left(K, \frac{x}{y}\right)$. It has the property that $x \cdot$ meridian $+y \cdot$ longitude of $K$ becomes contractible in $M\left(K, \frac{x}{y}\right)$. The same discussion holds for links instead of knots.

Now given a framed knot $(M, K, f)$. Then

$$
K^{\prime}: S^{1} \rightarrow 0 \times S^{1} \hookrightarrow D^{2} \times S^{1} \hookrightarrow M(K, f)
$$

is a knot depending only on $[f]$ with a canonical diffeomorphism $f: \partial \nu K^{\prime} \rightarrow$ $\partial \nu K$. Let $f^{\prime}$ be a framing for $K^{\prime}$.

Lemma 2.2 In the above situation there is a canonical diffeomorphism

$$
M(K,[f])\left(K^{\prime},\left[f^{\prime}\right]\right) \simeq M\left(K,\left[f \circ f^{\prime}\right]\right) .
$$

If $M$ is a homology 3-sphere, this specialises to

$$
M\left(K, \frac{1}{y}\right)\left(K^{\prime}, \frac{x^{\prime}}{y^{\prime}}\right) \simeq M\left(K, \frac{x^{\prime}}{x^{\prime} y+y^{\prime}}\right) .
$$


Proof. Let $\alpha \in \pi_{0} \operatorname{Diff}\left(D^{2} \times S^{1}\right)$. Consider the commutative diagram

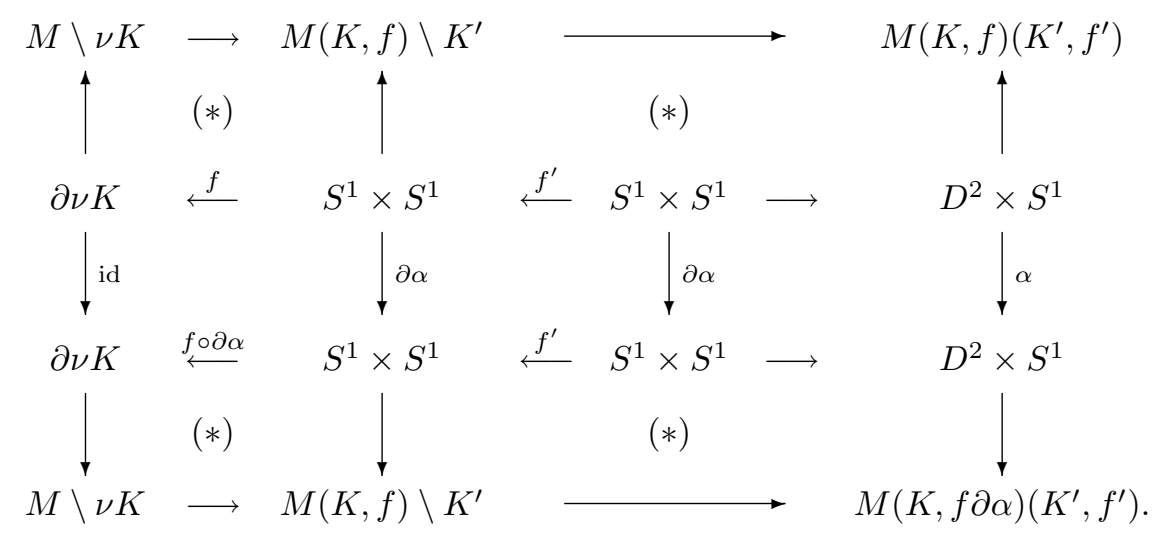

The squares marked with $(*)$ are colimit diagrams. By transitivity of colimit diagrams we have $M(K, f)\left(K^{\prime}, f^{\prime}\right) \simeq M\left(K, f f^{\prime}\right)$ and $M(K, f \partial \alpha)\left(K^{\prime}, f^{\prime}\right) \simeq$ $M\left(K, f(\partial \alpha) f^{\prime}\right)$. The above diagram induces a canonical diffeomorphism between $M(K, f)\left(K^{\prime}, f^{\prime}\right) \simeq M\left(K, f f^{\prime}\right)$ and $M(K, f \partial \alpha)\left(K^{\prime}, f^{\prime}\right)$. This proves the first statement.

If $M$ is a homology 3 -sphere, so is $M\left(K, \frac{1}{y}\right)$. Choose an orientation of $K$. Let $m, l$ be a meridian and longitude for $K$. With respect to the canonical basis we choose

$$
f:=\left(\begin{array}{ll}
1 & 0 \\
y & 1
\end{array}\right)
$$

$S^{1} \times 1$ is a meridian for $K^{\prime}$. A longitude for $K^{\prime}$ is given by $f^{-1}(l)$, which is $1 \times S^{1}$. Thus with respect to the canonical basis of $S^{1} \times S^{1}$ we choose

$$
f^{\prime}:=\left(\begin{array}{ll}
x^{\prime} & u^{\prime} \\
y^{\prime} & v^{\prime}
\end{array}\right),
$$

with $x^{\prime} v^{\prime}-y^{\prime} u^{\prime}=1$. Then

$$
f f^{\prime}=\left(\begin{array}{cc}
x^{\prime} & u^{\prime} \\
x^{\prime} y+y^{\prime} & y u^{\prime}+v^{\prime}
\end{array}\right)
$$

yielding $M\left(K, f f^{\prime}\right) \simeq M\left(K, \frac{x^{\prime}}{x^{\prime} y+y^{\prime}}\right)$.

Q.E.D.

Lemma 2.3 The oriented 3-manifold $S(a, b, m)$ is diffeomorphic to a Dehn surgery on a framed link associated to the $\mathbb{Q}$-weighted graph

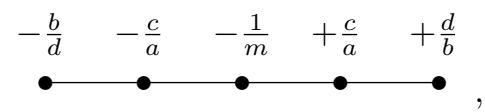

where $c, d \in \mathbb{Z}$ are chosen such that $a d-b c=1,0<d<a$ and $0<c<b$. 
Proof. Given $\left[s_{n}, \ldots, s_{1}\right]=\frac{\alpha}{\beta} \in \overline{\mathbb{Q}}, s_{i} \in \mathbb{Z}$. Assume $\beta^{*} \beta \equiv 1(\alpha)$ and $0<\beta^{*}<x$. Then $\left[-s_{n}, \ldots,-s_{1}\right]=-\left[s_{n}, \ldots, s_{1}\right]$ and $\left[s_{1}, \ldots s_{n}\right]=\frac{\alpha}{\beta^{*}}$, see [BPV, p. 81]. A fundamental rule of Kirby calculus [Ro, p. 272] says that framed links associated to the $\overline{\mathbb{Q}}$-weighted graphs

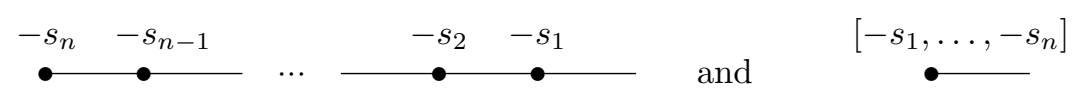

yield diffeomorphic 3-manifolds. The proof is an application of these three facts. Consider e.g. the branch in (2.1) labeled with $\frac{b}{b-x}$. Write $\frac{b}{b-x}=\left[s_{n}, \ldots, s_{1}\right]$. Then $-\left[s_{1}, \ldots, s_{n}\right]=\frac{-b}{(b-x)^{*}}$. Since $(b-x)^{*} \equiv-x^{*} \equiv a^{*} \bmod b$ we have $(b-x)^{*}=d$. This gives the vertex with the surgery weight $-\frac{b}{d}$. The other branches have to be handled similarly.

Q.E.D.

\section{$3 \quad S(a, b, m)$ is a Dehn surgery on a knot}

In this section we show that $S(a, b, m)$ is obtained by a rational surgery on a framed knot in $S^{3}$ and compute its Casson invariant.

Choose a framed link $L=L_{1} \cup L_{2}$ and a knot $K$ in $S^{3}$, associated to the following graph:

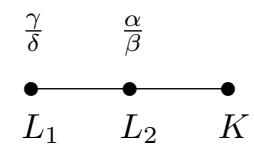

The knot $K$ induces a knot $K^{\prime}$ in $S^{3}(L)$.

Lemma 3.1 Assume $\alpha \gamma-\beta \delta=: \sigma \in\{ \pm 1\}$. Then $S^{3}(L)$ is diffeomorphic to $S^{3}$, and the knot $K^{\prime}$ in $S^{3}(L) \simeq S^{3}$ is the torus $k$ not $T(-\sigma \beta, \gamma)$. A longitude $l$ of $K$ in $S^{3}$ is $\sigma \gamma \beta \cdot$ meridian + longitude of $K^{\prime}$ in $S^{3}(L)$.

Proof. Orient the link $K \cup L$ such that all linking numbers are 1. Choose meridians $m_{1}, m_{2}$ and longitudes $l_{1}, l_{2}$ for $L_{1}, L_{2}$ respectively. Then $K, m_{2}, l_{1}$ are isotopic, as well as $m_{1}, l_{2}$. Define matrices in $\mathrm{SL}(2, \mathbb{Z})$ by

$$
A_{1}:=\left(\begin{array}{cc}
\gamma & \sigma \beta \\
\delta & \sigma \alpha
\end{array}\right), A_{2}:=\left(\begin{array}{cc}
\alpha & \sigma \delta \\
\beta & \sigma \gamma
\end{array}\right), A_{0}:=\left(\begin{array}{cc}
0 & 1 \\
1 & 0
\end{array}\right) .
$$

Then $S^{3}(L)$ is defined as a colimit of the diagram

$$
\begin{aligned}
& S^{3} \backslash \nu L \quad \hookleftarrow \quad \partial \nu L_{1} \stackrel{A_{1}}{\longleftarrow} S^{1} \times S^{1} \quad \hookrightarrow \quad D^{2} \times S^{1} \\
& S^{3} \backslash \nu L \quad \hookleftarrow \partial \nu L_{2} \quad \stackrel{A_{2}}{\longleftarrow} S^{1} \times S^{1} \quad \hookrightarrow \quad D^{2} \times S^{1} .
\end{aligned}
$$


Since $M \backslash \nu L$ is canonically diffeomorphic to $-\partial \nu L_{1} \times D^{1}$, there is a canonical isotopy between $\partial \nu L_{1}$ and $\partial \nu L_{2}$, giving the orientation reversing diffeomorphism $A_{0}: \partial \nu L_{1} \rightarrow \partial \nu L_{2}$.

Now $A_{1}^{-1} A_{0} A_{2}=\sigma A_{0}$, so $S^{3}(L)$ is diffeomorphic to a colimit of the diagram

$$
D^{2} \times S^{1} \hookleftarrow S^{1} \times S^{1} \stackrel{\sigma A_{0}}{\longleftarrow} S^{1} \times S^{1} \hookrightarrow D^{2} \times S^{1},
$$

which is a standard Heegaard splitting of genus 1 of $S^{3}$. Since $K$ is a meridian of $L_{2}$, and

$$
A_{2}^{-1}=\left(\begin{array}{cc}
\sigma \gamma & -\sigma \delta \\
-\beta & \alpha
\end{array}\right)
$$

one sees that $K^{\prime}$ is the torus $\operatorname{knot} T(-\beta, \sigma \gamma)=T(-\sigma \beta, \gamma)$.

Let $l$ be a longitude of $K$, and $l^{\prime}$ be the induced knot in $S^{3}(L)$. We have to compute the linking number $\mathrm{lk}\left(K^{\prime}, l^{\prime}\right)$. Let $B$ be the linking matrix of $L$, and define the $2 \times 1$-matrices $B_{K}:=\left(\operatorname{lk}\left(K, L_{1}\right), \operatorname{lk}\left(K, L_{2}\right)\right)^{t}, B_{l}:=$ $\left(\mathrm{lk}\left(l, L_{1}\right), \mathrm{lk}\left(l, L_{2}\right)\right)^{t}$. According to [Ho, lemma 1.1] one has

$$
\operatorname{lk}_{S^{3}(L)}\left(K^{\prime}, l^{\prime}\right)=\mathrm{lk}_{S^{3}}(K, l)-B_{K} B^{-1} B_{l}^{t}=\sigma \gamma \beta
$$

Thus $l^{\prime}$ is given by $\sigma \gamma \beta \cdot$ meridian + longitude of $K^{\prime}$.

Q.E.D.

Proposition 3.2 The oriented 3 -manifold $S(a, b, m)$ is obtained as the rational Dehn surgery with surgery weight $-\frac{1}{m}$ on the knot $T(a, b) \sharp T(-a, b)$, the connected sum of $T(a, b)$ and $T(-a, b)$.

Proof. Let $K$ be a framed knot in $S^{3}$ associated to the vertex in the middle of the graph in lemma 2.3 , and let $L_{-}, L_{+}$be framed links in $S^{3}$ coresponding to the left and the right part in the picture of the graph in lemma 2.3. Since $L_{-}$and $L_{+}$can be separated by an embedded 2-sphere in $S^{3}$, there is a diffeomorphism between $S^{3}\left(L_{-} \cup L_{+}\right)$and the connected sum $\tilde{S}^{3}:=S^{3}\left(L_{-}\right) \sharp S^{3}\left(L_{+}\right)$, which is diffeomorphic to $S^{3}$ by lemma 3.1. The framed knot $K$ induces a framed knot in $\tilde{S}^{3}$. The separating 2 -sphere splits $K=K_{-} \sharp K_{+}$as the connected sum of two unknots, so that $K^{\prime}=K_{-}^{\prime} \sharp K_{+}^{\prime}$. By lemma $3.1 K_{ \pm}$is the torus knot $T( \pm a, b)$.

To calculate the induced surgery weight in $\tilde{S}^{3}$, we choose a meridian $m$ of $K$ and longitudes $l, l_{-}, l_{+}$of $K, K_{-}, K_{+}$respectively, and let $l^{\prime}, m^{\prime}$ be the induced knots in $\tilde{S}^{3}$. Then $m^{\prime}$ is also a meridian for $K^{\prime}$. We show that $l^{\prime}$ is a longitude for $K^{\prime}$, too. We have to calculate $\operatorname{lk}\left(K^{\prime}, l^{\prime}\right)$. But

$$
\begin{aligned}
\operatorname{lk}_{S^{3}\left(L_{-} \cup L_{+}\right)}\left(K^{\prime}, l^{\prime}\right) & =\mathrm{lk}_{S^{3}\left(L_{-} \cup L_{+}\right)}\left(K_{-}^{\prime} \sharp K_{+}^{\prime}, l_{-}^{\prime} \sharp l_{+}^{\prime}\right) \\
& =\mathrm{lk}_{S^{3}\left(L_{-}\right)}\left(K_{-}^{\prime}, l_{-}^{\prime}\right)+\mathrm{l}_{S^{3}\left(L_{+}\right)}\left(K_{+}^{\prime}, l_{+}^{\prime}\right) \\
& =-a b+a b \\
& =0
\end{aligned}
$$


Thus $l^{\prime}$ is a longitude for $K^{\prime}$, so the surgery weight for $K^{\prime}$ is the same as for $K$, namely $-\frac{1}{m}$.

Q.E.D.

Corollary 3.3 The Casson invariant of $S(a, b, m)$ is

$$
\lambda(S(a, b, m))=-\frac{m\left(a^{2}-1\right)\left(b^{2}-1\right)}{6} .
$$

Proof. Define $T$ to be the torus knot $T(a, b)$, and $K:=T \sharp T^{*}$. By proposition 3.2 and the behaviour of Casson's invariant under surgery [Ak-MC] one inductively obtains

$$
\lambda(S(a, b, m))=\lambda\left(S^{3}\left(K,-\frac{1}{m}\right)\right)=m \lambda\left(S^{3}(K,-1)\right)
$$

On the other hand we can use the symmetrised Alexander polynomial $\Delta_{K}(t)$ $[\mathrm{Ak}-\mathrm{MC}]$ to compute

$$
\lambda\left(S^{3}(K,-1)\right)=\frac{1}{2} \frac{\partial^{2}}{\partial t^{2}}\left(\Delta_{T \sharp T^{*}}\right)(1)=-\frac{1}{2} \frac{\partial^{2}}{\partial t^{2}} \Delta_{T}^{2}(1),
$$

where the last equality holds since $\Delta$ is multiplicative under forming connected sums and changes sign under passing to the mirrored knot. Set $d:=$ $-\frac{1}{2}(a-1)(b-1)$. The symmetrised Alexander polynomial of the torus $\operatorname{knot} T$ is computed by [Ro, p. 178]

$$
\Delta_{T}(t)=\frac{\left(t^{a b}-1\right)(t-1)}{\left(t^{a}-1\right)\left(t^{b}-1\right)} \cdot t^{d}=\frac{\sum_{i=0}^{b-1} t^{a i+d}}{\sum_{j=0}^{b-1} t^{j}} .
$$

Calculating the first derivative of $\Delta_{T}$ one obtains

$$
\Delta_{T}^{\prime}(t)=\left(\sum_{j=0}^{b-1} t^{j}\right)^{-2} \sum_{i, j=0}^{b-1}(a i-j+d) t^{a i+j+d-1}
$$

One calculates from this $\Delta_{T}^{\prime}(1)=0$. Write $\Delta_{T}^{\prime}(t)=g(t)^{-1} f(t)$ as the quotient of two polynomials as above, then

$$
\begin{aligned}
\Delta_{T}^{\prime \prime}(1) & =g^{-2}(1)\left(f^{\prime}(1) g(1)-f(1) g^{\prime}(1)\right)=g^{-1}(1) f^{\prime}(1) \\
& =b^{-2} \sum_{i, j=0}^{b-1}(a i-j+d)(a i-j+d-1) .
\end{aligned}
$$

This expression is a quadratic polynomial in $a$. Since $\Delta_{T(a, b)}=\Delta_{T(a, b)^{*}}=$ $\Delta_{T(-a, b)}$ it is even. By the symmetry between $a$ and $b$ it is also an even, 
quadratic polynomial in $b$. It obviously vanishes for $b=1$, thus also for $b=-1$, and by symmetry also for $a= \pm 1$. Setting $a=0, b=2$, thus $d=\frac{1}{2}$, one obtains

$$
\frac{1}{4} \sum_{i, j=0}^{1}\left(\frac{1}{2}-j\right)\left(-\frac{1}{2}+j\right)=-1
$$

Thus $\Delta_{T}^{\prime \prime}(1)=\frac{1}{3}\left(a^{2}-1\right)\left(b^{2}-1\right)$.

Q.E.D.

\section{Characters in $\mathrm{SU}(2)$}

We will first determine the fundamental group of $S(a, b, m)$. For a graph $\Gamma$ denote by $\Gamma_{v}$ the set of vertices and by $\Gamma_{e}$ the set of edges.

Proposition 4.1 Let $\Gamma$ be a $\overline{\mathbb{Q}}$-weighted tree, $L$ be the associated framed link in $S^{3}$. For each vertex choose an ordering for its neighbours. Then $\pi_{1} S^{3}(L)$ admits a presentation with $\Gamma_{v}$ as the set of generators, and the following relations: Each vertex $v$ with weight $\frac{\alpha}{\beta}$ gives a relation

$$
v^{\alpha}\left(\prod_{\{v, w\} \in \Gamma_{e}} w\right)^{\beta}
$$

where the product runs according to the ordering of the neighbours. Each edge $\{v, w\} \in \Gamma_{e}$ gives a relation

$$
v w v^{-1} w^{-1}
$$

A generator $v \in \Gamma_{v}$ is represented by a meridian of the link component $L_{v}$.

Proof. Choose an orientation of $L$. Since $\Gamma$ is a tree, we can assume that all linking numbers are 1 . Let $X:=S^{3} \backslash L$.

Claim: $\pi_{1} X$ admits a presentation with a set of generators $\Gamma_{v}$ and a set of relations $\left\{v w v^{-1} w^{-1} \mid\{v, w\} \in \Gamma_{e}\right\}$. The generators $v \in \Gamma_{v}$ are represented by the meridians of the of the link components $L_{v}$.

We proof the claim by induction on the number $l$ of vertices. Without loss we may assume that $\Gamma$ is connected. The claim is trivially true for $l=0$ and $l=1$. For $l=2$ the claim is also true, since $X$ is homotopy equivalent to the 2 -torus. Assume $l>2$. Since $\Gamma$ is a tree, there is a bud $v$. Let $\Gamma^{\prime}$ be the tree obtained by removing $v$. Let $S$ be a Seifert surface for $L_{v}$. Define $X_{1}$ as $X \backslash S$ and $X_{2}$ as the intersection of $\mathrm{X}$ with a regular neighborhood of $S$ in $S^{3}$. Then the 1-point compactification of $X_{2}$ is homeomorphic to the complement of the Hopf link. $X_{1}$ is diffeomorphic to the link complement of $\Gamma^{\prime}$. Now the induction hypothesis, together with the theorem of Seifert and van Kampen, completes the induction. 
Note that by our choice of orientation of $L$ the homotopy class of a longitude of a component $L_{v}$ is represented by the product over the meridians of all $L_{w}$, where $w$ is a neighbour of $v$. The proof of the proposition is completed by another application of the theorem of Seifert and van Kampen.

Q.E.D.

As an immediate consequence we obtain from lemma 2.3

Corollary 4.2 The fundamental group $\pi_{1} S(a, b, m)$ admits a presentation with generators

$$
x, \mu, \omega, \eta, u
$$

and relations

$$
x^{-b} \mu^{d}, \quad \mu^{-c}(x \omega)^{a}, \omega^{-1}(\mu \eta)^{m}, \eta^{c}(\omega u)^{a}, u^{d} \eta^{b}
$$

together with the commutator relations corresponding to the edges of the graph in lemma 2.3.

Given $a=\left(a_{1}, \ldots, a_{n}\right) \in \mathbb{Z}^{n}$, denote by $T(a)$ the triangle group

$$
\left\langle X_{1}, \ldots, X_{n} \mid X_{1}^{a_{1}}, \ldots, X_{n}^{a_{n}}, \prod X_{i}\right\rangle,
$$

and by $\Sigma(a)$ the Seifert fibre space $\left\{\left(z_{1}, \ldots, z_{n}\right) \in \mathbb{C}^{n} \mid \sum z_{i}^{a_{i}}=0\right\} \cap S^{2 n-1}$. If the $a_{i}$ are pairwise relatively prime, this is a homology 3 -sphere. Its fundamental group is isomorphic to

$$
\left.\left\langle X_{1}, \ldots, X_{n}, Z\right| Z \text { central, } X_{i}^{a_{i}} Z^{b_{i}}, \prod X_{i}\right\rangle,
$$

where the $b_{i} \in \mathbb{Z}$ are chosen such that $1=\sum \frac{b_{i}}{a_{i}} \prod a_{i}$. Give $\Sigma(a)$ the orientation such that $\left(\left(a_{1}, b_{1}\right), \ldots,\left(a_{n}, b_{n}\right)\right)$ is a sequence of Seifert invariants for $\Sigma(a)$. Denote by Rep and Rep* the space of representations and nontrivial representations respectively, by $\chi$ and $\chi^{*}$ the space of characters and nontrivial characters respectively.

Proposition 4.3 The following representation spaces are canonically isomorphic:

(i). $\operatorname{Rep}^{*}\left(\pi_{1} S(a, b, m), \mathrm{SU}(2)\right)$

(ii). $\operatorname{Rep}^{*}\left(\pi_{1} S(a, b, m), \mathrm{SO}(3)\right)$

(iii). $\operatorname{Rep}^{*}(T(a, b, m a b-1), \mathrm{SO}(3)) \cup \operatorname{Rep} *(T(a, b, m a b+1), \mathrm{SO}(3))$

(iv). $\operatorname{Rep}^{*}\left(\pi_{1} \Sigma(a, b, m a b-1), \mathrm{SU}(2)\right) \cup \operatorname{Rep}^{*}\left(\pi_{1} \Sigma(a, b, m a b+1), \mathrm{SU}(2)\right)$

(v). $\operatorname{Rep} *\left(\pi_{1} \Sigma(a, b, m a b-1), \mathrm{SO}(3)\right) \cup \operatorname{Rep} *\left(\pi_{1} \Sigma(a, b, m a b+1), \mathrm{SO}(3)\right)$ 
Proof. Let $S$ be a $\mathbb{Z} / 2$-homology 3-sphere, $\rho: \pi_{1} S \rightarrow \mathrm{SO}(3)$ a representation, then $\rho$ lifts uniquely to $\mathrm{SU}(2)$ : Let $u: S \rightarrow B\left(\pi_{1} S\right) \approx K\left(\pi_{1} S, 1\right)$ classify the universal covering of $S$, then $\pi_{1} u$ is bijective, $\pi_{2} u$ is surjective, thus by the Whitehead theorem $H_{1}(u)$ is also bijective, $H_{2}(u)$ is surjective. It follows that $H^{2}\left(\pi_{1} S, \mathbb{Z} / 2\right) \simeq H^{2}\left(K\left(\pi_{1} S, 1\right), \mathbb{Z} / 2\right)=0$. Thus all central $\mathbb{Z} / 2$-extension of $\pi_{1} S$ are split. Since also $H^{1}\left(\pi_{1} S, \mathbb{Z} / 2\right)=0$, such a splitting is unique. Especially all $\mathrm{SO}(3)$-representations admit a unique lift. This is the canonical isomorphisms between (i) and (ii), and (iv) and (v).

We will show the isomorphism between (ii) and (iii). Dint of eigenspaces, the following is true: Given elements $a, b, c$ in $\mathrm{SU}(2)$ such that $a$ commutes with $b, b$ commutes with $c$, and $b$ is non-central. Then $a$ commutes with $c$. Since $\rho$ admits a $\mathrm{SU}(2)$-lift, the same property holds in $\operatorname{im} \rho$.

Let $\rho \in \operatorname{Rep}^{*}\left(\pi_{1}(S(a, b, m), \mathrm{SO}(3))\right.$. We will identify elements in $\pi_{1} S(a, b, m)$ with their images in $\mathrm{SO}(3)$. We will look at $\mu, \omega$ and $\eta$. The following four cases are possible:

Case $\mu, \omega, \eta \neq 1$. Then $\rho$ is abelian, and by the theorem of Hurewicz $\rho$ factors over $H_{1}(S(a, b, m))$, hence is trivial. Contradiction.

Case $\omega=1$. We have the relations $x^{-b} \mu^{d}, \mu^{-c} x^{a}$ and $\eta^{c} u^{a}, u^{d} \eta^{b}$. Since $a d-b c=1$ the relations $x, \mu, \eta, u$ follow. Thus $\rho$ is trivial, a contradiction.

Case $\mu=1$. We have the relations $x^{-b},(x \omega)^{a}$. $(\omega, \eta, v)$ generate an abelian subgroup with presentation matrix

$$
\left(\begin{array}{ccc}
-1 & b & 0 \\
m & -d & c \\
0 & b & -a
\end{array}\right)
$$

Calculating its minors one sees that it is cyclic of order $m a b-1$ with generator $\omega$. Thus these representations correspond bijectively to representations of $T(a, b, m a b-1)$.

Case $\eta=1$. This case is symmetric to the case $\mu=1$. The representations correspond bijectively to representations of $T(a, b, m a b+1)$.

The isomorphism between (iv) and (iii) is immediate, since every element in $\operatorname{Rep}^{*}\left(\pi_{1} \Sigma(a, b, m a b \pm 1), \mathrm{SO}(3)\right)$ has to send the central generator to $1 . \quad$ Q.E.D.

Corollary 4.4 The real analytic space $\chi^{*}(S(a, b, m), \mathrm{SU}(2))$ is discret and smooth.

Proof. By [Fi-St, Prop. 2.3] the topological spaces $\chi^{*}(\Sigma(a, b, m a b \pm 1), \mathrm{SU}(2))$ are discret, hence also $\chi^{*}(S(a, b, m), \mathrm{SU}(2))$. Let $[\rho] \in \chi^{*}(S(a, b, m), \mathrm{SU}(2))$ and denote by $\left[\rho_{T}\right],\left[\rho_{\Sigma}\right]$ the corresponding characters on $T(a, b, m a b \pm 1)$ and $\Sigma(a, b, m a b \pm 1)$. The vector space $\operatorname{su}(2)$ becomes a module over $\pi_{1} S(a, b, m)$ and $\pi_{1} \Sigma(a, b, m a b \pm 1)$ via ad $\circ \rho$ and ad $\circ \rho_{\Sigma}$. By [Fi-St, Prop. 2.5]

$$
H^{1}\left(\pi_{1} \Sigma(a, b, \operatorname{mab} \pm 1), \mathrm{su}(2)\right)=0 .
$$


Define the group $K$ by the extension

$$
1 \rightarrow K \rightarrow \pi_{1} \Sigma(a, b, m a b \pm 1) \rightarrow T(a, b, m a b \pm 1) \rightarrow 1 .
$$

Then su(2) viewed as a $K$-module is trivial. Consequently, the Hochschild-Serre spectral sequence

$$
\left.H^{p}\left(T(a, b, m a b \pm 1), H^{q}(K, \mathrm{su}(2))\right) \Longrightarrow H^{p+q}\left(\pi_{1} \Sigma(a, b, m a b \pm 1), \mathrm{su}(2)\right)\right)
$$

collapses, yielding in particular an isomorphism

$$
H^{1}\left(\pi_{1} \Sigma(a, b, m a b \pm 1), \mathrm{su}(2)\right)=H^{1}(T(a, b, m a b \pm 1), \mathrm{su}(2)) .
$$

The same argument shows that $H^{1}\left(\pi_{1} S(a, b, m), \mathrm{su}(2)\right)=H^{1}(T(a, b, m a b \pm$ $1), \mathrm{su}(2))$. It follows

$$
H^{1}\left(\pi_{1} S(a, b, m), \operatorname{su}(2)\right)=0 .
$$

But this is the tangent space of $[\rho]$, see $[\mathrm{Lu}-\mathrm{Ma}]$. Thus $[\rho]$ is a smooth point of $\chi^{*}(S(a, b, m), \mathrm{SU}(2))$.

Q.E.D.

We now compare the character spaces $\chi(\Sigma(a, b, m a b \pm 1), \mathrm{SU}(2))$. Let $T$ be the standard maximal torus in $\mathrm{SU}(2)$ and $W \simeq \mathbb{Z} / 2$ be its Weyl group. The mapping

$$
r:=\frac{1}{2} \text { trace }: \mathrm{SU}(2) \longrightarrow[0, \pi] \simeq(\mathbb{R} / 2 \pi \mathbb{Z}) / W \simeq T / W
$$

is a quotient for the conjugacy operation. Thus the mapping

$$
\begin{gathered}
l^{ \pm}: \chi(\Sigma(a, b, m a b \pm 1), \mathrm{SU}(2)) \longrightarrow \mathbb{Z}^{3}, \\
\left.\rho \mapsto\left(r\left(\rho\left(X_{1}\right)\right) \frac{a}{\pi}, r\left(\rho\left(X_{2}\right)\right) \frac{b}{\pi}, r\left(\rho\left(X_{3}\right)\right) \frac{m a b \pm 1}{\pi}\right)\right),
\end{gathered}
$$

associating to a character its rotation vector, is injective.

Proposition 4.5 There is a bijection of sets

$$
\psi: \chi(\Sigma(a, b, m a b-1), \mathrm{SU}(2)) \rightarrow \chi(\Sigma(a, b, m a b+1), \mathrm{SU}(2)) .
$$

If $a, b$ are odd, then $\psi$ can be chosen such that for all characters $[\rho] \in \chi(\Sigma(a, b$, mab1), $\mathrm{SU}(2))$ one has

$$
l^{-}([\rho])=\left(l_{1}, l_{2}, l_{3}\right) \Longrightarrow l^{+}(\psi([\rho]))=\left(l_{1}, l_{2}, l_{3}+1\right) .
$$

Proof. Let $\left[\rho^{-}\right] \in \chi(\Sigma(a, b, m a b-1))$ with $r\left(\left[\rho^{-}\right]\right)=:\left(l_{1}, l_{2}, l_{3}^{-}\right)$Choose $\rho^{-} \in\left[\rho^{-}\right]$such that $x:=\rho^{-}\left(X_{1}\right) \in T$. Let $C$ be the conjugacy class of $\rho^{-}\left(X_{2}\right)$. Since $\rho$ is nontrivial it follows that $x$ is noncentral, $C$ is diffeomorphic to $S^{2}$, and $C \cap T$ consists of two different points $\left\{p_{1}, p_{2}\right\}$. Consequently $I:=r(x C) \subset[0, \pi]$ is a proper, closed interval. We claim that $Y:=r^{-1}\left(x p_{i}\right) \cap C$ is discrete. Given 
$c, c^{\prime} \in C$ such that $y:=x c, y^{\prime}:=x c^{\prime} \in Y$ are conjugate to $x p_{i}$ by $g, g^{\prime} \in \mathrm{SU}(2)$. Then $x p_{i} \in g T g^{-1} \cap g^{\prime} T g^{\prime-1}$. Since $x p_{i}$ is noncentral, these are the same tori [Br-tD, Thm. 2.11], i.e. $g^{-1} g^{\prime} \in N T$. It follows that $x c=\left(x p_{i}\right)^{ \pm 1}$. The same holds for $x c^{\prime}$, which proves the claim. Consequently $\left\{r\left(x p_{1}\right), r\left(x p_{2}\right)\right\}=\partial I$, since otherwise the connected space $x C \backslash Y$ would have to be disconnected.

In our case $\partial I$ is represented by the two numbers $\frac{l_{1} b \pm l_{2} a}{a b} \pi$, consequently the lenght of $I$ is an even multiple of $\frac{\pi}{a b}$. Thus one can write $I$ as the union of nonoverlapping intervals of the form $\left[\frac{x}{m a b} \pi, \frac{x+2}{m a b} \pi\right]=: I_{x}, x \in \mathbb{Z}, 0 \leq x \leq$ $m a b-2$. By the elementary inequalities

$$
\begin{aligned}
& \frac{x}{m a b+1}<\frac{x}{m a b}<\frac{x+1}{m a b+1}<\frac{x+1}{m a b}<\frac{x+2}{m a b+1} \\
& \frac{x-1}{m a b-1}<\frac{x}{m a b}<\frac{x+1}{m a b-1}<\frac{x+1}{m a b-1}
\end{aligned}
$$

it follows that each $I_{x}$ contains exactly two elements of the form $\frac{x}{m a b-1}, \frac{x+1}{m a b-1}$ and $\frac{x+1}{m a b+1}, \frac{x+2}{m a b+1}$. Choose an $x$ such that $I_{x}$ contains $\frac{l_{3}^{-}}{m a b-1}$. Now choose $l_{3}^{+}$ as the unique element such that $\frac{l_{3}^{+}}{m a b+1} \in I_{x}$ and $(-1)^{l_{3}^{+}}=\rho(Z)$.

By construction, $\left(l_{1}, l_{2}, l_{3}^{+}\right)$gives a well-defined a $\mathrm{SU}(2)$-character $\left[\rho^{+}\right]$of $\pi_{1} \Sigma(a, b, m a b+1)$. Define $\psi\left(\left[\rho^{-}\right]\right):=\left[\rho^{+}\right]$. Also by construction, this map is bijective.

If $a, b$ are odd, one might choose Seifert invariants $\left(a, b_{1}\right),\left(b, b_{2}\right)$ for the Sefeirt fibred spaces such that $b_{1}, b_{2}$ are even. Then also $l_{1}, l_{2}$ have to be even. Choose $l_{3}^{+}$such that $\frac{l_{3}^{+}}{m a b+1} \in I_{x}$ and $l_{3}^{+} \equiv h+1 \bmod 2$. Then there is a $\left[\rho^{+}\right] \in \chi(\Sigma(a, b, m a b+1), \mathrm{SU}(2))$ with $\rho^{+}(H)=-\rho^{-}(H)$ and rotation vector $\left(l_{1}, l_{2}, l_{3}^{+}\right)$. Define $\psi\left(\left[\rho^{-}\right]\right)=\left[\rho^{+}\right]$. By construction, $\psi$ is also bijective and has the required properties.

Q.E.D.

\section{$5 \quad$ Bordisms to Seifert fibred spaces}

We now give a topological explanation of proposition 4.3 using a result of [KKR].

Let $K$ is a knot in $S^{3}$, and assume that $M$ is a homology 3-sphere with an embedded torus $T \hookrightarrow M$, yielding a decomposition $M \simeq M_{1} \cup_{T} M_{2}$, such that $M_{1}$ is diffeomorphic to $S^{3} \backslash \nu K$. Denote by $i: M_{1} \rightarrow M$ the inclusion. In [KKR] a bordism $B$ between $M$ and another closed 3-manifold $M^{\prime}$ is constructed such that the bordism decomposes along $T \times I \hookrightarrow B$. This embedding splits the borism into two connected components $B=B_{1} \cup_{T \times I} B_{2}$. Here $B_{2}$ is equal to $M_{2} \times I$. In their construction $\partial B_{1}$ is diffeomorphic to $S^{3}(K, 0)$, the 0 -surgery on $K$, by the decomposition $\partial B_{1}=M_{1} \cup_{T \times 0} T \times I \cup_{T \times 1} D^{2} \times S^{1}$.

The bordism $B$ has the property for each representation $\rho: \pi_{1} M \rightarrow \mathrm{SU}(2)$ such that $i^{*} \rho$ is abelian there are well defined representations $\rho^{\prime}: \pi_{1} M^{\prime} \rightarrow \mathrm{SU}(2)$ and $\beta: \pi_{1} B \rightarrow \mathrm{SU}(2)$ such that $\partial \beta=\rho \amalg \rho^{\prime}$. 
Let $V_{K}$ be a Seifert matrix for $K$, and define a matrix

$$
B_{K}(t):=(1-t) V_{K}+\left(1-t^{-1}\right) V_{K}^{t} .
$$

It is related to the symmetrised Alexander matrix $A_{K}(t):=t^{\frac{1}{2}} V-t^{-\frac{1}{2}} V^{t}$ for $V_{K}$ by

$$
B_{K}(t)=\left(t^{-\frac{1}{2}}-t^{\frac{1}{2}}\right) A_{K}(t) .
$$

[KKR, Thm. 4.4] implies:

Proposition 5.2 Let $\mu$ be a meridian for $K$. Let $\rho$ be an $\mathrm{SU}(2)$-representation of $\pi_{1} M$ such that $i^{*} \rho$ is abelian. Define $\theta \in[0, \pi]$ by requiring that $\rho(\mu)$ is conjugate to

$$
\left(\begin{array}{cc}
e^{i \theta} & \\
& e^{-i \theta}
\end{array}\right) \in \mathrm{SU}(2)
$$

and assume that $e^{2 i \theta}$ is not a root of the Alexander polynomial of $K$. Then the Floer gradings of $\rho, \rho^{\prime}$ are related by

$$
\operatorname{grad}_{M}(\rho) \equiv \operatorname{grad}_{M^{\prime}}\left(\rho^{\prime}\right)-\operatorname{Sign} B_{K}\left(e^{2 i \theta}\right) \bmod 8 .
$$

Since by (5.1) the function $t \mapsto \operatorname{Sign} B_{K}(t)$ is constant on the unit circle away from the roots of the Alexander polynomial. At the roots of the Alexander polynomial it clearly can jump only by an even number. Since $\operatorname{Sign} B_{K}(1)=0$ it follows

$$
\operatorname{grad}_{M}(\rho) \equiv \operatorname{grad}_{M^{\prime}}\left(\rho^{\prime}\right) \bmod 2 .
$$

We will apply this to two different toral decompositions of $M:=S(a, b, m)$. The following picture defines two tori $T_{\sigma} \hookrightarrow S(a, b, m)$, for $\sigma \in\{ \pm 1\}$ :

Please insert figure 1 here.

Let $M_{\sigma, 1}$ be the component of $S(a, b, m)$, in which the reader is located. By lemma 3.1 the $M_{\sigma, 1}$ is diffeomorphic to the complement of a torus knot $T(\sigma a, b)$, and the 0 -framing of the torus knot corresponds to $\sigma a b$-meridian + longitude in the above picture. Thus $M_{\sigma}^{\prime}$ is given by Dehn surgery according to the weighted graph

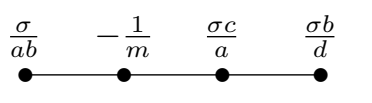


Now use Kirby calculus to expand the vertex with weight $-\frac{1}{m}$ and then contract the vertex with weight $\frac{\sigma}{a b}$. After one further contraction we end up with the graph

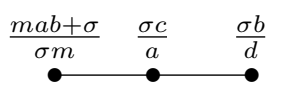

But this Dehn surgery yields the Seifert fibred space $\Sigma(a, b, m a b+\sigma)$. The meridian of the knot complement in $M_{1}$ is the generator $\omega$ in corollary 4.2, so the representation $\rho^{\prime}$ is the representation canonically associated to $\rho$ in proposition 4.3. This gives:

Corollary 5.3 Let $\rho \in \chi(S(a, b, m), \mathrm{SU}(2))$ be of type $\sigma \in\{ \pm 1\}$. Then

$$
\operatorname{grad}_{S(a, b, m)}(\rho)=\operatorname{grad}_{\Sigma(a, b, m a b+\sigma)}\left(\rho^{\prime}\right)-\operatorname{Sign} B_{T(\sigma a, b)}\left(e^{2 \pi i l_{3}^{\sigma}\left(\rho^{\prime}\right)}\right) .
$$

We also can compute the underlying abelian group of $\operatorname{HF}(S(a, b, m))$ :

Corollary 5.4 The Floer comlex of $S(a, b, m)$ lies in the even part of the grading. Consequently all differentials vanish, and $\operatorname{HF}(S(a, b, m))$ is a free abelian group of rank $\frac{1}{3} m\left(a^{2}-1\right)\left(b^{2}-1\right)$, lying in the even part of the grading.

Proof. By [Fi-St] the Floer grading on the Seifert fibre spaces is even. By corollary 5.3 and the remark after proposition 5.2 this is also true for $S(a, b, m)$. By [Ta] the Casson invariant, calculated in corollary 3.3 , is $-\frac{1}{2}$ times the Euler characteristic of the Floer homology.

Q.E.D.

In [Fi-St] there is a formula for the Floer grading on the Seifert fibre spaces, so in principle the Floer grading on $S(a, b, m)$ can be computed.

Given $x, y \in \mathbb{R}, n \in \mathbb{Z}$ such that $n \neq 0$, define the real number

$$
\Upsilon(n, x, y):=-\frac{2}{n} \sum_{k=1}^{n-1} \cot \left(\frac{x \pi k}{n}\right) \cot \left(\frac{\pi k}{n}\right) \sin ^{2}\left(\frac{y \pi k}{n}\right) .
$$

Define the integer $\Delta(x, y)$ as the weighted sum over the lattice points in the triangle $\Delta$ in $\mathbb{R}^{2}$ with vertices given by $(0,0),(x, 0),(0, y)$, according to the following weight: A lattice point $(n, m)$ is counted with the weight whose signum is $\operatorname{Sign}(n m)$ and whose absolute value is $0,1,2$ or 4 , according to wether $(n, m)$ is $(0,0)$, a vertex $\neq(0,0)$, lies on an edge but is not a vertex or lies in the interiour of $\Delta$.

By [Fi-St, Thm. 3.9] the Floer grading for a representation $\rho$ on $\Sigma\left(a_{1}, \ldots, a_{n}\right)$ with rotation vector $\left(l_{1}, \ldots, l_{n}\right)$ is given by

$$
\operatorname{grad}_{\Sigma\left(a_{1}, \ldots, a_{n}\right)}(\rho)=-\frac{2 e^{2}}{A}-3+\sum_{i=1}^{n} \Upsilon\left(a_{i}, \frac{A}{a_{i}}, \frac{A}{a_{i}} l_{i}\right),
$$


where $e:=\sum \frac{A}{a_{i}} l_{i}$ and $A:=\prod a_{i}$. Assuming that $n, x, y \in \mathbb{Z}, n, x \neq 0$ and $x \mid y$ there is the combinatorial formula [Ca-Go, p. 188]

$$
\Upsilon(n, x, y)=\frac{2 y^{2}}{x n}-\Delta\left(\frac{y}{n}, \frac{y}{x}\right) .
$$

Proposition 5.7 Assume $a, b$ odd. Given $\sigma \in\{ \pm 1\}$. Let $\rho$ be a SU(2)character on $\Sigma(a, b$, mab $+\sigma)$ with rotation vector $\left(l_{1}, l_{2}, l_{3}\right)$. Then the Floer grading of $\rho$ is

$$
\operatorname{grad}_{\Sigma(a, b, m a b+\sigma)}(\rho) \equiv-\Delta\left(l_{3}, \frac{a b}{m a b+\sigma} l_{3}\right)-\sigma \Delta\left(l_{2}, \frac{a}{b} l_{2}\right)-\sigma \Delta\left(l_{1}, \frac{b}{a} l_{1}\right)-3
$$

modulo 8 .

Proof. One calculates

$$
\frac{A}{a^{2}} \equiv \sigma \frac{b}{a}, \quad \frac{A}{b^{2}} \equiv \sigma \frac{a}{b}, \quad \frac{A}{(m a b+\sigma)^{2}} \equiv \frac{a b}{m a b+\sigma} \quad \bmod 1
$$

and

$$
-\frac{2 e^{2}}{A}=-\frac{2 \sigma b l_{1}^{2}}{a}-\frac{2 \sigma a l_{2}^{2}}{b}-\frac{2 a b l_{3}^{2}}{m a b+\sigma}-2 m\left(b l_{1}+a l_{2}\right)^{2}-4\left(b l_{1}+a l_{2}\right) l_{3} .
$$

Since $a, b$ are odd we can choose Seifert invariants for $\Sigma(a, b, m a b+\sigma)$ such that $l_{1}, l_{2}$ have to be even. Then

$$
-\frac{2 e^{2}}{A} \equiv-\frac{2 \sigma b l_{1}^{2}}{a}-\frac{2 \sigma a l_{2}^{2}}{b}-\frac{2 a b l_{3}^{2}}{m a b+\sigma} \bmod 8 .
$$

Putting this into (5.5) and using (5.6) finishes the proof.

Q.E.D.

Now define the $\mathbb{Z} / 4$-graded Floer homology $\mathrm{HF}_{\mathbf{Z} / 4}$ as $\mathrm{HF}$, with the grading induced by $\mathbb{Z} / 8 \rightarrow \mathbb{Z} / 4$.

Proposition 5.8 Assume $a, b$ odd. Then the Floer groups $\operatorname{HF}_{\mathbf{Z} / 4}(S(a, b, 1))$ are 2-periodic.

Proof. By proposition 4.3 the generators of $\operatorname{HF}_{\mathbf{z} / 4}(S(a, b, 1))$ come in pairs $\left(\rho^{-}, \rho^{+}\right)$, where $\rho^{-}$is of type -1 and $\rho^{+}=\psi\left(\rho^{-}\right)$. Then the rotation vectors of corresponding characters on the Seifert fibred spaces, also called $\rho^{ \pm}$, are of the form $\left(l_{1}, l_{2}, l_{3}\right),\left(l_{1}, l_{2}, l_{3}+1\right)$ with $0<l_{3}<m a b-1$. We have to compute the difference between the grading and signature term in 5.3 modulo 4. After proposition 5.7 the difference of the Floer gradings is

$$
\operatorname{grad}_{\Sigma(a, b, a b+1)}\left(\rho^{+}\right)-\operatorname{grad}_{\Sigma(a, b, a b-1)}\left(\rho^{-}\right)
$$




$$
\begin{aligned}
& \equiv-\Delta\left(l_{3}+1, \frac{a b}{a b+1}\left(l_{3}+1\right)\right)+\Delta\left(l_{3}, \frac{a b}{a b-1} l_{3}\right) \\
& \equiv-1-2\left(l_{3}+1-1+\left[\frac{a b}{a b+1}\left(l_{3}+1\right)\right]\right)+1+2\left(l_{3}-1+\left[\frac{a b}{a b-1} l_{3}\right]\right) \\
& \equiv 2\left(l_{3}+l_{3}\right)+2\left(l_{3}-1+l_{3}\right) \\
& \equiv 2 \bmod 4 .
\end{aligned}
$$

On the other hand the difference of the signature terms is

$$
\begin{aligned}
& -\operatorname{Sign} B_{T(a, b)}\left(e^{\frac{2 \pi i\left(l_{3}+1\right)}{a b+1}}\right)+\operatorname{Sign} B_{T(a, b)^{*}}\left(e^{\frac{2 \pi i l_{3}}{a b-1}}\right) \\
& =-\operatorname{Sign} B_{T(a, b)}\left(e^{\frac{2 \pi i\left(l_{3}+1\right)}{a b+1}}\right)-\operatorname{Sign} B_{T(a, b)}\left(e^{\frac{2 \pi i l_{3}}{a b-1}}\right) .
\end{aligned}
$$

The roots of the symmetrised Alexander polynomial for $T(a, b)$ are contained in the $a b$-th roots of unity. By the inequalities

$$
\frac{l_{3}}{a b}<\frac{l_{3}+1}{a b+1}<\frac{l_{3}}{a b-1}<\frac{l_{3}+1}{a b}
$$

the segment $e^{2 \pi i\left[\frac{l_{3}+1}{a b+1}, \frac{l_{3}}{a b-1}\right]}$ does not contain any $a b$-th roots of unity. Thus $\operatorname{Sign} B_{T(a, b)}(t)$ is constant on that segment. It has to be even. Thus

$$
\begin{aligned}
-\operatorname{Sign} B_{T(a, b)}\left(e^{\frac{2 \pi i\left(l_{3}+1\right)}{a b+1}}\right)-\operatorname{Sign} B_{T(a, b)}\left(e^{\frac{2 \pi i l_{3}}{a b-1}}\right) & \equiv 2 \operatorname{Sign} B_{T(a, b)}\left(e^{\frac{2 \pi i l_{3}}{a b-1}}\right) \\
& \equiv 0 \bmod 4 .
\end{aligned}
$$

Consequently,

$$
\begin{aligned}
& \operatorname{grad}_{S(a, b, 1)}\left(\rho^{+}\right)-\operatorname{grad}_{S(a, b, 1)}\left(\rho^{-}\right) \\
& \quad \equiv \operatorname{grad}_{\Sigma(a, b, a b+1)}\left(\rho^{+}\right)-\operatorname{grad}_{\Sigma(a, b, a b-1)}\left(\rho^{-}\right) \\
& \quad \equiv 2 \bmod 4 .
\end{aligned}
$$

This means that $\operatorname{HF}_{\mathbf{z} / 4}(S(a, b, 1))$ is 2-periodic.

Q.E.D.

\section{Floer's Triangle}

We will use Floer's triangle to proof inductively a structure theorem on the Floer homology $\operatorname{HF}(S(a, b, m))$.

For each complement $\Sigma \backslash K$ of a knot $K$ in a homology 3-sphere $\Sigma$, Floer has defined a 4-periodic, affinely $\mathbb{Z} / 8$-graded abelian group $\operatorname{HF}(K)=\operatorname{HF}(\Sigma \backslash K)$ $[\mathrm{Fl}],[\mathrm{Br}-\mathrm{Do}]$, lying in an exact triangle

$$
\begin{array}{lll} 
& \operatorname{HF}(K) & \\
& & \nwarrow \\
\mathrm{HF}(\Sigma) & \longrightarrow & \mathrm{HF}(K,-1)),
\end{array}
$$

where the maps respect the underlying affine grading. 
Theorem 6.1 The Floer group $\operatorname{HF}(S(a, b, m))$ is a free abelian group of rank $\frac{1}{3} m\left(a^{2}-1\right)\left(b^{2}-1\right)$, lying in the even part of the grading. It is 4-periodic. If $a, b$ are odd it is 2-periodic.

Proof. The statement on the underlying abelian group is proposition $5.4 \mathrm{We}$ proof the periodicity by induction on $m$. Since $S(a, b, 0) \simeq S^{3}$ the theorem is true for $m=0$. Define the knot $K:=T(a, b) \sharp T(a, b)^{*}$ in $S^{3}$. Recall $S(a, b, m) \simeq$ $S^{3}\left(K,-\frac{1}{m}\right)$. Since HF $\left(S^{3}\right)=0$, for $m=1$ Floer's exact triangle reduces to an isomorphism

$$
0 \rightarrow \mathrm{HF}(S(a, b, 1)) \rightarrow \mathrm{HF}(K) \rightarrow 0,
$$

so $\operatorname{HF}(K) \simeq \operatorname{HF}\left(S(a, b, 1)\right.$ is 4-periodic. If $a, b$ are odd, then $H_{\mathbf{Z} / 4}(S(a, b, 1)$ is 2-periodic by proposition 5.8, consequently $\operatorname{HF}(K) \simeq \operatorname{HF}(S(a, b, 1)$ has to be 2-periodic. Assume inductively that the periodicity property is proven for $m-1$. By lemma 2.2 the knot $K \hookrightarrow S^{3}$ defines a knot $K^{\prime} \hookrightarrow S(a, b, m-1)$ such that $S(a, b, m-1) \backslash K^{\prime} \simeq S^{3} \backslash K$ and $S(a, b, m) \simeq S(a, b, m-1)\left(K^{\prime},-1\right)$. Now Floer's exact triangle reads

$$
\mathrm{HF}(S(a, b, m-1)) \quad \longrightarrow \quad \begin{aligned}
& \mathrm{HF}(K) \\
& \mathrm{HF}(S(a, b, m)) .
\end{aligned}
$$

By proposition 5.4

$$
\operatorname{rank} \operatorname{HF}(S(a, b, m))=\operatorname{rank} \operatorname{HF}(S(a, b, m-1))+\operatorname{rank} \operatorname{HF}(S(a, b, 1)),
$$

so the above exact triangle has to split into a short exact sequence

$$
0 \rightarrow \operatorname{HF}(S(a, b, m-1)) \rightarrow \operatorname{HF}(S(a, b, m)) \rightarrow \operatorname{HF}(K) \rightarrow 0 .
$$

By induction hypothesis the groups on the left and the right are 4-periodic, and 2-periodic if $a, b$ are odd. Thus the same holds for $\operatorname{HF}(S(a, b, m))$, completing the induction.

Q.E.D.

\section{References}

[Ak-MC] Akbulut, S., McCarthy, J.D.: Casson's invariant for homology 3sheres. (Princeton Math. Notes 36) Princeton: Princeton University Press 1990.

[BPV] Barth, W., Peters, C., Van de Ven, A: Compact complex surfaces. (Ergeb. Math. Grenzgeb., 3. Folge Bd. 4) Berlin Heidelberg New York Tokyo: Springer 1984.

[Br-Do] Braam, P.J., Donaldson, S.K.: Floer's work on instanton homology and knots. Preprint. 
[Br-tD] Bröcker, T., tom Dieck, T.: Representations of compact Lie groups. (Grad. Texts Math., Vol. 98) Berlin Heidelberg New York Tokyo: Springer 1985.

[Ca-Go] Casson, A., Gordon, C.: Cobordism of classical knots. In: Guillou, L., Marin, A.: A la recherche de la topologie perdue. (Prog. Math., Vol. 62) Boston Basel Stuttgart: Birkhäuser 1986.

[tD-Pe] tom Dieck, T., Petrie, T.: Contractible affine surfaces of Kodeira dimension one. Jap. J. Math. 16, No. 1, 147-169, (1990).

[Fi-St] Fintushel, R., Stern, R.: Instanton homology of Seifert fibred homology three spheres. Proc. London Math. Soc. (3) 61 (1990), 109-137.

[Fl] Floer, A: Instanton homology, surgery and knots. In: Donaldson, S.K., Thomas, C.B.: Geometry of low-dimensional manifold: 1. (Lond. Math. Soc. Lect. Note Ser. 150) Cambridge, Cambridge University Press 1992.

[Ho] Hoste, J.: A formula for Casson's invariant. Trans. Am. Math. Soc. 297, Vol. 2, 547-562 (1986).

[KKR] Kirk, P., Klassen, E., Ruberman, D.: Splitting the spectral flow and the Alexander matrix. Preprint.

[Lu-Ma] Lubotzky, A., Magid, A.: Varieties of group representations of finitely generated groups. Memoirs of the AMS, Vol. 58, No. 336 (1985).

[Ra] Ramanujam, C.P.: A topological characterisation of the affine plane as an algebraic variety. Ann. of Math. 94, 68-88. (1971)

[Ro] Rolfsen, D.: Knots and links. Houston: Publish or Perish 1990.

[St] Stern, R.: Computing Donaldson Invariants. Lectures for the Park City Geometry Institute, July 1994.

[St-Sz] Stipsicz, A., Szabó, Z.: Floer homology groups of certain algebraic links. Preprint.

[Ta] C.H. Taubes: Casson's invariant and gauge theory. J. Diff. Geom. 31, 547-599 (1990). 
Stefan Schröer

Universität Bielefeld

Fachbereich Mathematik, SFB 343

Postfach 100131

33501 Bielefeld, Germany

Tel 49 (0)521 106 4973, Fax 49 (0)521 1064743

E-Mail schroeer@mathematik.uni-bielefeld.de 